\title{
A study on coherent structures and drag-reduction in the wall turbulence with polymer additives by TRPIV
}

\author{
Xin-Lei Guan · Shi-Yong Yao · Nan Jiang
}

Received: 4 January 2013 / Revised: 24 February 2013 / Accepted: 8 April 2013

(C)The Chinese Society of Theoretical and Applied Mechanics and Springer-Verlag Berlin Heidelberg 2013

\begin{abstract}
An experimental measurement was performed using time-resolved particle image velocimetry (TRPIV) to investigate the spatial topological character of coherent structures in wall-bounded turbulence of polymer additive solution. The fully developed near-wall turbulent flow fields with and without polymer additives at the same Reynolds number were measured by TRPIV in a water channel. The comparisons of turbulent statistics confirm that due to viscoelastic structure of long-chain polymers, the wall-normal velocity fluctuation and Reynolds shear stress in the near-wall region are suppressed significantly. Furthermore, it is noted that such a behavior of polymers is closely related to the decease of the motion of the second and forth quadrants, i.e., the ejection and sweep events, in the near-wall region. The spatial topological mode of coherent structures during bursts has been extracted by the new mu-level criteria based on locally averaged velocity structure function. Although the general shapes of coherent structures are unchanged by polymer ad-
\end{abstract}

The project was supported by the National Natural Science Foundation of China (11272233), National Key Basic Research and Development Program (2012CB720101) and 2012 opening subjects of The State Key Laboratory of Nonlinear Mechanics (LNM), Institute of Mechanics, Chinese Academy of Sciences.

X.-L. Guan · S.-Y. Yao · N. Jiang

Department of Mechanics,

Tianjin University, 300072 Tianjin, China

N. Jiang (函)

Tianjin Key Laboratory of Modern Engineering Mechanics, 300072 Tianjin, China

The State Key Laboratory of Nonlinear Mechanics, Institute of Mechanics, Chinese Academy of Sciences, 100190 Beijing, China

Nankai University-Tianjin University Center

for Liu Hui Applied Mathematics, 300072 Tianjin, China

e-mail: nanj@tju.edu.cn ditives, the fluctuating velocity, velocity gradient, velocity strain rate and vorticity of coherent structures during burst events are suppressed in the polymer additive solution compared with that in water. The results show that due to the polymer additives the occurrence and intensity of coherent structures are suppressed, leading to drag reduction.

Keywords Time-resolved particle image velocimetry · Wallbounded turbulence - Coherent structures - Polymer additives $\cdot$ Drag reduction

\section{Introduction}

Dissolving a small amount of long-chain, high-molecularweight polymers to turbulent wall-bounded flow can lead to dramatic drag reduction. This phenomenon was first discovered by Toms [1] and has received considerable attention afterwards because of the increasing importance of drag reduction in modern fluid engineering applications. For instance in oil pipelines, only tens p.p.m. ( $\mathrm{mg} / \mathrm{kg}$ ) of polymer solution might lead to significant reduction of power consumption and increase of the flow rate of oil, so that drag reduction with additives has a wide range of applications in the field of crude oil and oil products transportation. Although this effect has been discovered for over half a century, a thorough understanding of the mechanism in which polymers reduce drag has not yet been clearly revealed. During the past six decades, a vast number of experimental papers have been published about polymeric drag reduction. As for turbulence statistics theory, the measurement work of Virk et al. [2] in a pipe flow should be mentioned. They found that there was an asymptotic value for the maximum drag reduction, which is independent of polymers and pipe diameter. The phenomenon in the regions where drag reduction occurs with polymer additives were also discussed by Lumley [3] and Reischman and Tiederman [4] who presented experimental evidences that the buffer region changed and played an important role in drag-reducing flows. Measurements in chan- 
nel flow with polymer injection, using two-component laserDoppler velocimetry (LDV) were performed by Luchik and Tiederman [5] and Walker and Tiederman [6]. They reported that the addition of polymers caused a decrease in wall normal velocity fluctuations and a concomitant reduction of wall shear stress, which indicated a damping of energy exchange between near-wall region and outer region. More recently, the result of Ptasinski et al. [7] and Oppong et al. [8] showed that the Reynolds stress strongly decreased and the stress generated by viscoelasticity became dominant instead of Reynolds stress in the total stress. With respect to coherent structure, the first paper addressing the effect of polymers on coherent structure was written by Donohue et al. [9] who employed color dye flow visualization to examine the coherent structure in wall turbulence. They reported a sharp decline of $y$-fluid motions close to the wall, and an increase in the streak spacing and a significant decrease in bursting rate. Achia and Thompson [10] used real-time holography to visually examine the structure of wall turbulence in a pipe flow. They reported that the polymer additives suppressed the formation of streaks and the occurrence of bursts. Warholic et al. [11] focused on the influence of drag-reducing polymers on the coherent structure in a turbulent channel flow. They indicated that polymer additives caused a decrease of smallscale structure, as evidenced by the decline in contributions of high-wave numbers to the power spectra. More recently, Motozawa et al. [12] presented channel flow experimental investigation with polymer solution injection, using particle image velocimetry (PIV). The instantaneous velocity vector field suggested that the intensity and frequency of ejections and sweeps in the near-wall region both decreased with polymer additives.

Conditional sampling is an important method to identify coherent structure in wall-bounded turbulence. Luchik and Tiederman [5] employed mu-level method (modified ulevel method) on the coherent structures in turbulent channel flows for the LDV dataset to discuss the effects of polymers on the streak spacing, bursting frequency, and Reynolds stress. The mu-level method was employed by Luchik and Tiederman [13], and its detection function $D(t)$ for the leading edge is defined by

$D(t)= \begin{cases}1, & u \leqslant-L u^{\prime}, \\ 0, & u \geqslant-0.25 u^{\prime},\end{cases}$

where $L$ is the threshold level in the range of $0.5-1.3$. As Eq. (1) shows, an event will be detected if the coherent structures pass through the measurement point at which the instantaneous velocity is much smaller than the mean velocity. It means that the low-speed fluid near the wall ejects outward to reach the measurement point, leading to the gradual decrease of the local velocity with time. Thus the mulevel method could effectively identify the ejection events. However, there are two shortcomings in conditional sampling method. One is the subjectivity due to the preselected threshold and the other is that small-scale fluctuation might affect the detection results. To remedy the shortcomings, Jiang et al. [14] proposed the concept of locally averaged velocity structure function to describe the local deformation and relative motion of turbulent eddy structures within definite scale. One-dimensional locally averaged velocity structure function is given by

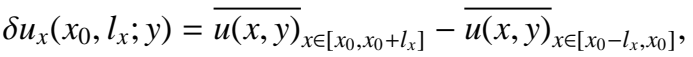

where $l_{x}$ donates the spatial scale in the streamwise direction. $\delta u_{x}\left(x_{0}, l_{x} ; y\right)$ may be interpreted as the relative difference between locally averaged velocities in the two ranges of $x \in\left[x_{0}, x_{0}+l_{x}\right]$ and $x \in\left[x_{0}-l_{x}, x_{0}\right]$, respectively. For the coherent structure, the case $\delta u_{x}\left(x_{0}, l_{x} ; y\right)>0$ represents the stretching process within an eddy due to the upstream averaged convective velocity being smaller than the downstream one while the case $\delta u_{x}\left(x_{0}, l_{x} ; y\right)<0$ exhibits the compressing process within an eddy owing to the upstream averaged convective velocity being larger than the downstream one. The mu-level method searches for those regions where the low-speed fluid ejects outward from the wall, in which case the local streamwise fluctuating velocity reaches the negative minimum instantaneously, i.e., the low-pass filtered first derivative reaches the zero point from negative to positive. Thus, the fact that the locally averaged velocity structure function reaches the zero point from negative to positive could be used to detect the point where the streamwise fluctuating velocity reaches the local negative minimum instantaneously. Correspondingly, for the case of high velocity fluid inrushing toward the wall, the low-pass filtered first derivative reaches the zero point from positive to negative. Consequently, the detection criteria for coherent structures in wall turbulence based on the locally averaged velocity structure function is defined as

$$
D\left(x_{0}, l_{x} ; y_{0}\right)= \begin{cases}1 \text { (ejection), } & \text { if } \delta u_{x}\left(x_{0}-\Delta x, l_{x} ; y_{0}\right)<0, \\ & \delta u_{x}\left(x_{0}+\Delta x, l_{x} ; y_{0}\right)>0, \\ -1 \text { (sweep), } & \text { if } \delta u_{x}\left(x_{0}-\Delta x, l_{x} ; y_{0}\right)>0, \\ & \delta u_{x}\left(x_{0}+\Delta x, l_{x} ; y_{0}\right)<0, \\ 0, & \text { otherwise, }\end{cases}
$$

where $\Delta x$ is the grid size in the streamwise direction. Overall, the new mu-level method not only makes a breakthrough with regard to the limitation of preselected threshold, but also increases the accuracy in identifying burst events (sweeps). Furthermore, as the turbulent motion has an inherent characteristic of multi-scale [15], the concept of local average within a local range with a definite scale is supposed to be involved in burst events detection scheme to eliminate the effect of small-scale fluctuations on the accuracy of detection results. With the multi-scale property, the new mu-level method is more reasonable and appropriate for the purpose of investigating the coherent structures in turbulent flows with or without polymer additive solution.

In the present paper, the influence of polymer additives on the mean flow field of wall-bounded turbulence will 
be investigated by analyzing turbulence statistics, including streamwise mean velocity profile, turbulence intensities, Reynolds shear stress and turbulent kinetic energy. Moreover, the new mu-level method will be employed to investigate the mechanism of drag reduction by polymer additives from the viewpoint of coherent structure modification. Since the coherent structures are dominant structures in the turbulent boundary layer, it is essential to investigate the turbulent drag-reduction with polymers from the viewpoint of coherent structures control. Indeed, several studies in this aspect have been performed, but none of them have obtained accurate spatial topologies of coherent structures. Since the new mu-level method involves the scale parameter, more accurate results on the drag-reducing mechanism of polymers can be revealed. The spatial topologies of fluctuating velocity, velocity gradients, vorticity and velocity strain rate during ejections and sweeps, are extracted using spatial phase-averaged method, which is expressed by

$$
\begin{aligned}
& \langle f(x, y)\rangle_{\mathrm{e}}=\frac{1}{N} \sum_{k=1}^{N} f\left(x_{0}(k)+x, y_{0}(k)+y\right), \\
& x \in\left[-\frac{l_{x}}{2}, \frac{l_{x}}{2}\right], \quad y \in\left[-\frac{l_{y}}{2}, \frac{l_{y}}{2}\right], \\
& D\left(x_{0}(k), l_{x} ; y_{0}(k)\right)=1, \\
& \langle f(x, y)\rangle_{\mathrm{s}}=\frac{1}{M} \sum_{k=1}^{M} f\left(x_{0}(k)+x, y_{0}(k)+y\right), \\
& x \in\left[-\frac{l_{x}}{2}, \frac{l_{x}}{2}\right], \quad y \in\left[-\frac{l_{y}}{2}, \frac{l_{y}}{2}\right], \\
& D\left(x_{0}(k), l_{x} ; y_{0}(k)\right)=-1,
\end{aligned}
$$

where $f(x, y)$ represents the turbulent characteristic quantity such as the fluctuating velocity or vorticity, and $\left(x_{0}(k), y_{0}(k)\right)$ indicates the spatial location where a $k$-th burst occurs, \langle\rangle represents the ensemble average of certain variables. Based on the detection of burst events, the mechanism of polymer drag reduction will be discussed from the viewpoint of energy cascade of turbulence.

\section{Experiment description}

The experiment was carried out in an open circulating water channel with a test section of $1200 \mathrm{~mm} \times 140 \mathrm{~mm} \times 150 \mathrm{~mm}$ (length $\times$ width $\times$ depth). With a 4:1 elliptical leading edge, a $1050 \mathrm{~mm}$ long, $138 \mathrm{~mm}$ wide and $10 \mathrm{~mm}$ thick flat acrylic glass plate was horizontally mounted on the bottom surface of the channel at a free-stream velocity of $0.18 \mathrm{~m} / \mathrm{s}$. A trip wire was employed at the leading edge to ensure fully developed turbulence at measurement location.

A 2D time-resolved particle image velocimetry (2DTRPIV) system was composed of double-cavity Nd: YAG lasers and a high speed camera $(1280 \times 1024$ pixels $)$. A schematic of the test system is shown in Fig. 1, where $x, y$, $z$ are the coordinates in the flow direction, in the direction perpendicular to the wall and in the spanwise direction. As the tracer particles, hollow glass microspheres with a median diameter of $10 \mu \mathrm{m}$ and density of $1.03 \mathrm{~g} / \mathrm{mm}^{3}$ were seeded in the turbulent flows. A laser sheet with a thickness of about $0.8 \mathrm{~mm}$ illuminated the flow measurement from the top of the channel. The laser plane lay on the axial center-plane of the channel along the streamwise direction. 2D-TRPIV measurement field was located at $0.9 \mathrm{~m}$ downstream of the leading edge in the $(x, y)$-plane. The high-speed camera acquired the particle images of instantaneous flow field from the side view. The present sampling frequency of the flow image was $600 \mathrm{~Hz}$. 6400 snapshots of particle images were recorded for the measured field with and without polymers respectively, and were analyzed in the following steps: adaptive correlation was carried out with interrogation windows of $32 \times 32$ pixels and an overlap rate of $75 \%$, range validation and the last step of average filter. The measured field is about $90.71 \mathrm{~mm} \times 72.55 \mathrm{~mm}$ (streamwise length $\times$ wallnormal height), consisting of $157 \times 125$ two-dimensional velocity vectors.

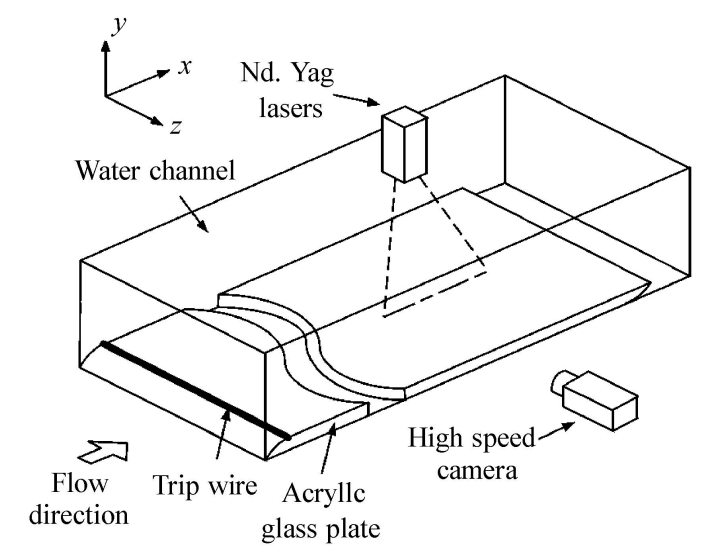

Fig. 1 Schematic diagram of the experimental set-up

The polymer chosen in the experiment was analytically pure polyacrylamide (PAM) with a molecular weight of 5 million. The polyacrylamide has a good anti-shear performance among the commonly used polymers for applications. As Kenis [16] observed, after a few tens of passes through turbulent flow, the polyacrylamide solution had the best friction reduction effect, compared with several other sorts of polymer solution, such as polyethylene oxide (PEO). The polymer solution was prepared by gradually dissolving the pure polyacrylamide in water and stirring constantly. The polymer solution was initially mixed to 16600 p.p.m. based on weight calculation. This concentrated mixture was allowed to hydrate for hours and then diluted to 190 p.p.m. in a tank with dials described by Cai [17] who analyzed the drag reduction effect of polyacrylamide solution at different concentrations. Then, the viscosity of polymer solution was checked by the use of a Brookfield LVDV-II + Pro viscometer with a ULA assembly at $50 \mathrm{rpm}$ (revolution per minute). The detailed data for the drag-reducing flow and the water flow are listed in Table 1. 
Table 1 Detailed data for the drag-reducing flow and the water flow

\begin{tabular}{lllllllll}
\hline & $R e_{\delta}$ & $\delta / \mathrm{mm}$ & $\mu /(\mathrm{mPa} \cdot \mathrm{s})$ & $u^{*} /\left(\mathrm{mm} \cdot \mathrm{s}^{-1}\right)$ & $\tau_{\mathrm{w}} /\left(\mathrm{kg} \cdot \mathrm{m}^{-1} \cdot \mathrm{s}^{-2}\right)$ & $c_{\mathrm{f}} / 10^{-3}$ & $D R / \%$ & Plotter symbol \\
\hline Solution & 4047 & 23.83 & 1.060 & 9.559 & 0.091 .216 & 5.640 & 16.58 & $\square$ \\
Water & 4062 & 22.70 & 1.006 & 10.466 & 0.109336 & 6.761 & - & $\circ$ \\
\hline
\end{tabular}

\section{Results}

\subsection{Turbulent statistics}

From Table 1, it is noted that for drag-reducing solution the thickness of turbulent boundary layer gets a bit thicker and the friction velocity is decreased compared with the case of water. The Reynolds number is based on the boundary layer thickness $\delta$ and freestream velocity $U_{0} . \quad \tau_{\mathrm{w}}$ and $c_{\mathrm{f}}$ represent wall shear stress and friction coefficient, respectively, defined as follows

$\tau_{\mathrm{w}}=\rho u^{* 2}, \quad c_{\mathrm{f}}=\frac{2 \tau_{\mathrm{w}}}{\rho U_{0}^{2}}=\frac{2 u^{* 2}}{U_{0}^{2}}$.

The amount of drag reduction is defined as

$D R=\frac{\tau_{\text {water }}-\tau_{\text {solution }}}{\tau_{\text {water }}}$.

The symbols appearing in the last column are consistently used in the following plots.

The mean streamwise velocity profiles for the dragreducing and the water flows at the same Reynolds number are presented in Fig. 2. The mean velocity profile in the logarithmic sublayer is given by $u^{+}=A \ln y^{+}+B$, in which the superscript " + " indicates that variable is nondimensionalized using the friction velocity and kinematic viscosity. $A, B$ and friction velocity are calculated by the Newton iterative and steepest descent method [18], whose details can be found in Fan and Jiang [19]. The measured mean velocity profiles, no matter with or without polymers, shown in Fig. 2 are both in agreement with the log-law. The mean velocity profile in the solution has the same slope as that in the water flow, which is consistent with the result of Warholic et al. [20]. They indicated that when the drag reduction rate was less than $35 \%$,

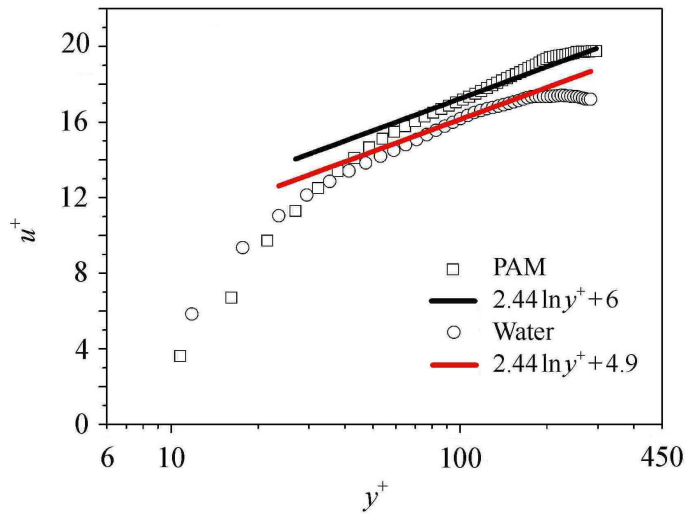

Fig. 2 Longitudinal mean velocity profiles of the turbulent boundary layer the slope of dimensionless mean velocity profile with polymers remained unchanged compared with the water case. However, the $B$ value of the solution becomes larger due to the polymers. The difference indicates that the buffer region becomes thicker, resulting in an additional lift in the logarithmic overlap region due to the existence of polymer additives. This effect reflects the character of drag reduction at the wall, and has also been observed by others [20-22].

Figure 3 compares the root mean square (r.m.s.) of the streamwise velocity component $u^{\prime}$ non-dimensionalized with the mean velocity for the solution and water at the same Reynolds number. It is shown that the shapes of the profiles of ${u^{\prime}}^{+}$are similar for both flows, but the peak value is shifted away from the wall to a higher $y^{+}$value for the polymer solution. Note that the peak levels of $u^{\prime} / U_{0}$ for the flows with equal Reynolds number are roughly the same. Trends in our experimental data are in agreement with those for the PIV data of Whiter et al. [23] and Motozawa et al. [12]. However, the peak values of $u^{\prime} / U_{0}$ for the drag-reducing flows in their papers were slightly higher than that for water flows. This difference in the peak value is attributed to the difference between the mean velocity and the friction velocity used in the nondimensionalization. This reason is consistent with Warholic et al. [11], who reported that due to the decrease in the friction velocities with polymer additives, the dimensional fluctuating streamwise velocities for the solutions were found to be approximately equal to those for water over a large region close to the wall.

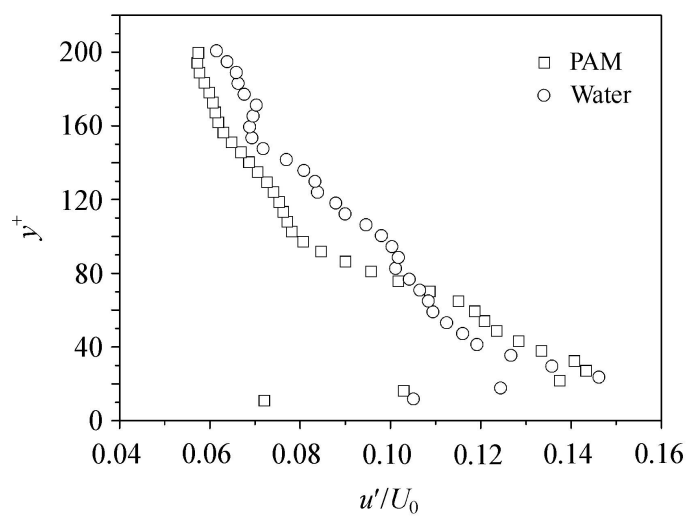

Fig. 3 Comparison of root mean square of streamwise velocity fluctuations

The r.m.s. of the wall-normal fluctuating velocity $v^{\prime}$ non-dimensionalized with the mean velocity is plotted in Fig. 4. Comparison with the water case shows that the peak 
in $v^{\prime} / U_{0}$ broadens as well as shifts further away from the wall when drag reduction occurs. A considerable decrease of $v^{\prime}$ can be noted for the drag-reducing flow. Close to the wall, at a given value of $y^{+}$, the values of $v^{\prime} / U_{0}$ for the drag-reducing solution are nearly no more than a half of those in the water. Clearly, the effect of polymers on the wall-normal motions is quite dramatic in the near wall region.

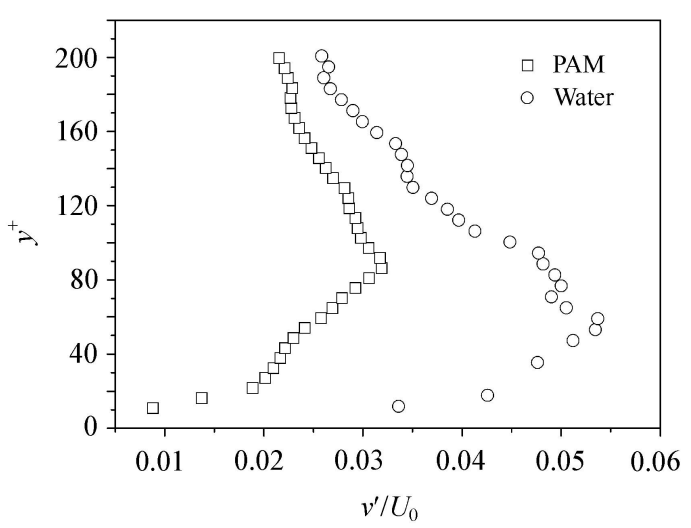

Fig. 4 Comparison of root mean square of wall-normal velocity fluctuations

Figure 5 shows the profiles of Reynolds shear stress normalized by $U_{0}^{2}$ with and without polymers. In water flow, Reynolds shear stress has a distinct peak near the wall. For polymer additive solution, the peak of the Reynolds shear stress significantly decreases and shifts a little away from the wall. The reduction of Reynolds shear stress means that the exchange of momentum weakens, and the turbulent fluctuation is also suppressed. In the outer region of the flow, the value of normalized Reynolds shear stress for the water is almost the same as that for the solution at the same Reynolds number. The difference in the behavior of polymers for different regions suggests that the solution suppresses the turbulent motion by weakening the burst of coherent structures. Meanwhile, this also confirms that coherent structures' bursting is the main mechanism for the maintenance, evolution and development of the turbulence.

The scatter plots of streamwise and wall-normal velocity fluctuations normalized by the friction velocity in the water flow and the polymer solution are presented in Fig. 6. Among these figures, Figs. $6 \mathrm{a}$ and $6 \mathrm{~b}$ are respectively obtained at $y^{+}=22$ and 20 in the buffer region, while another two are respectively obtained at $y^{+}=100$ and 99 in the logarithmic region. In water as shown in Fig. 6b, the scatter plot depicts the shape of a symmetrical ellipse whose major axis is inclined to the coordinate axes with a certain angle. The scatter points in the second $\left(u^{\prime}<0, v^{\prime}>0\right)$ and forth $\left(u^{\prime}>0, v^{\prime}<0\right)$ quadrants are much more than that in the first and third ones, which means that in turbulent flow the Reynolds shear stress is mainly produced by ejections $\left(u^{\prime}<0, v^{\prime}>0\right)$ and sweeps $\left(u^{\prime}>0, v^{\prime}<0\right)$. On the contrary, in the solution as shown in Fig. 6a, the elliptical shape is elongated with its two ends sharpened and the major axis becomes more parallel to the $u^{\prime}$-axis. The absolute value of $u^{\prime}-v^{\prime}$ product dramatically decreases in the second and fourth quadrants, indicating that ejections and sweeps decrease for polymer additives near the wall. Additionally, the magnitudes of streamwise velocity fluctuations change little while wall-normal velocity fluctuations sharply decline, which is consistent with the plots of Figs. 3 and 4. However, obviously the scatter plots are similar in Fig. $6 \mathrm{c}$ for the polymer solution and Fig. 6d for water in the logarithmic region, which suggests that polymer additives have little effect on turbulent structure in this area. As is noted, velocity fluctuations diminish in Figs. $6 \mathrm{c}$ and $6 \mathrm{~d}$ compared with Figs. $6 \mathrm{a}$ and $6 \mathrm{~b}$, because the near-wall region, especially the buffer layer, is the most active field of coherent structures burst in turbulent boundary layer.

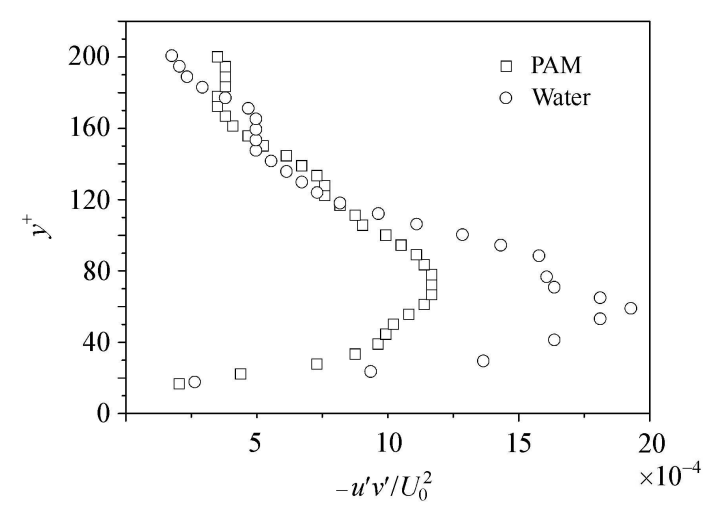

Fig. 5 Comparison of Reynolds shear stress profiles

Mean kinetic energy in the turbulent flow at scale $l_{x}$ obtained through locally averaged velocity structure function is calculated using

$$
\begin{aligned}
& E\left(l_{x} ; y\right)=\left\langle\left|\delta u_{x}\left(x_{0}, l_{x} ; y\right)\right|^{2}\right\rangle_{x_{0}} \\
& =\left\langle\left|\frac{1}{\sqrt{l_{x}}}\left[\int_{x_{0}}^{x_{0}+l_{x}} u(x, y) \mathrm{d} x-\int_{x_{0}-l_{x}}^{x_{0}} u(x, y) \mathrm{d} x\right]\right|^{2}\right\rangle_{x_{0}},
\end{aligned}
$$

where $l_{x}$ denotes the given scale. Liu et al. [24] indicated that coherent structures occupy most kinetic energy in the turbulent boundary layer through a comparison of the turbulent kinetic energy before and after the excluding of coherent structures. The distributions of mean kinetic energy at different scales for the drag reducing flow and the water flow are plotted in Figs. $7 \mathrm{a}$ and $7 \mathrm{~b}$, respectively. The $n$-th scale means that the scale parameter $l_{x}$ along the streamwise is equal to $2^{n} \Delta x$, where $\Delta x=0.567 \mathrm{~mm}$. As seen from the two figures, the largest values of turbulent kinetic energy occur at nearly $y^{+}=20$, confirming that buffer layer is the most active field of coherent structures burst. The other important feature of Fig. 7 is that the kinetic energy values for the solution sharply decrease compared with that for water. This trend implies that with polymer additives the burst intensity rapidly declines. 

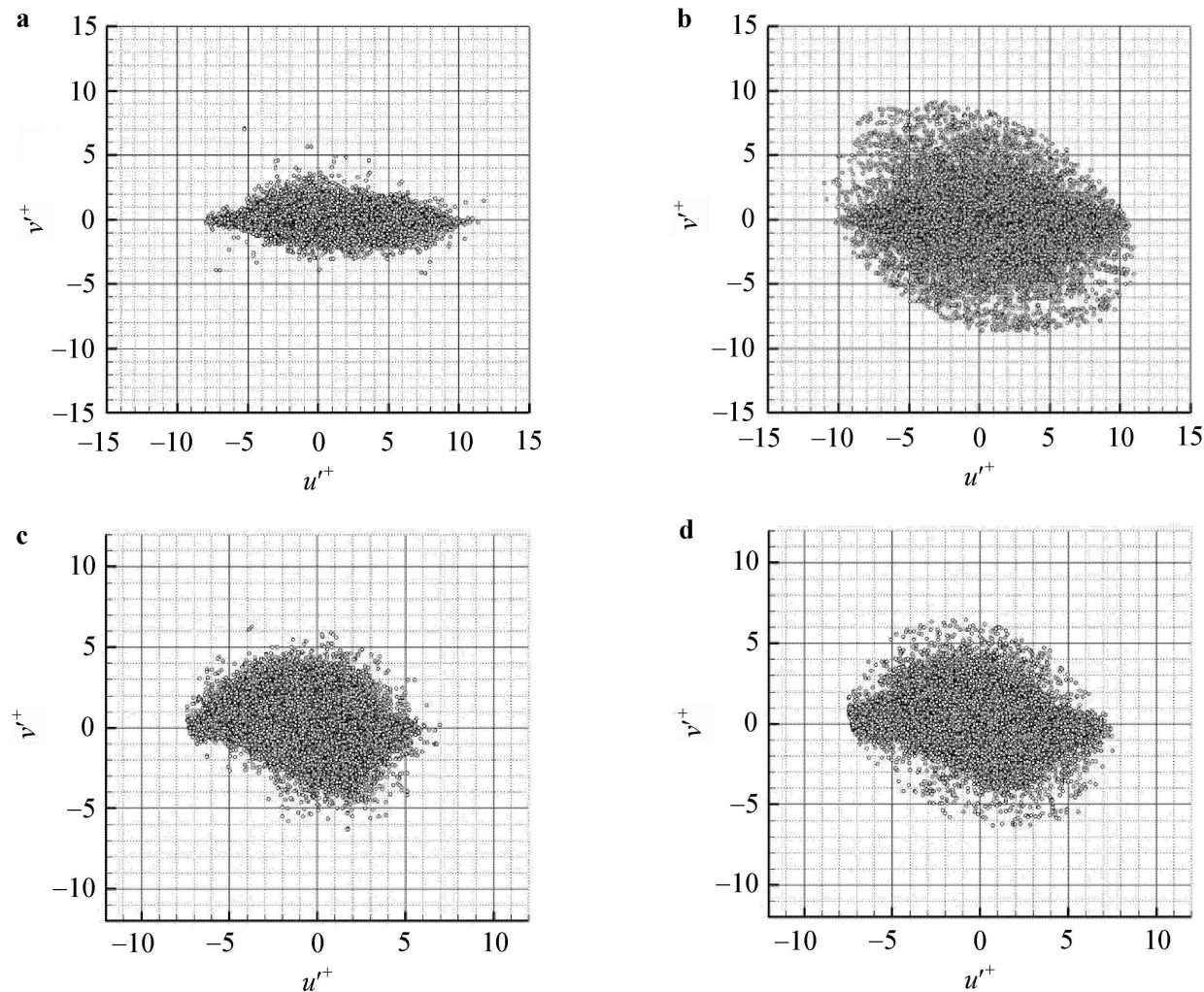

Fig. 6 Scatter plot of streamwise and wall-normal velocity fluctuations. a $y^{+}=22$ for PAM; $\mathbf{b} y^{+}=20$ for water; $\mathbf{c} y^{+}=100$ for PAM; d $y^{+}=99$ for water
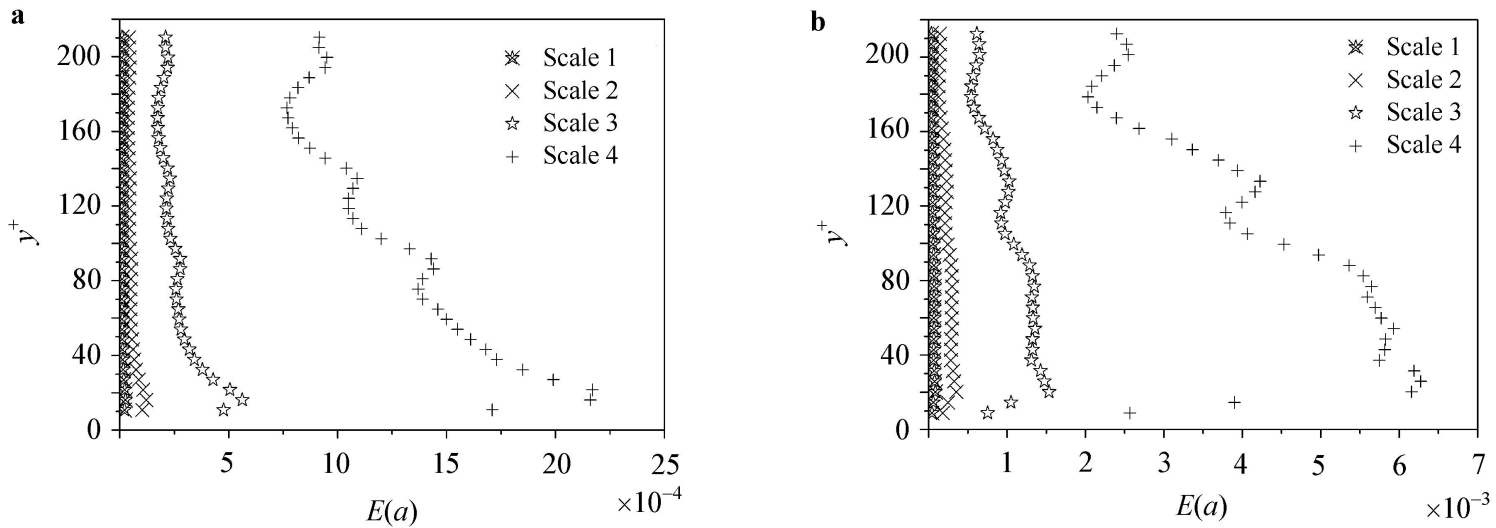

Fig. 7 Distributions of kinetic energy in the turbulent flows. a PAM; b Water

\subsection{Spatial topologic character of coherent structures}

The original fluctuating velocity components of turbulent boundary layer with and without polymers additives have both been decomposed into four scales by spatial local averaged velocity structure function. According to the maximum energy criterion, only the fourth scale, corresponding to $9.078 \mathrm{~mm}$ ( $\sim 16$ grids), is analyzed. The spatial topologies of coherent structures, in a detection area of $16 \times 32$ grids, have been obtained by the new detection criteria introduced in the first part, with the detecting center at $y^{+}=122$.
The contours of streamwise fluctuating velocity for the polymer solution and water during ejection are respectively shown in Fig. 8, where the flow direction is from left to right. A striking feature of this figure is that lying in the middle, low-speed fluids are surrounded by high-speed fluids. Besides, the low-speed fluids move away from the wall, in despite of the vectors appearing parallel to the wall, as the wall normal fluctuating velocities are nearly one order of magnitude smaller than the longitudinal ones. The phenomenon that the low-speed fluids eject outward from the wall is consistent with the feature of ejection event. Com- 
pared with the case of water, the distribution of longitudinal fluctuating velocity in the drag-reducing flow appears more

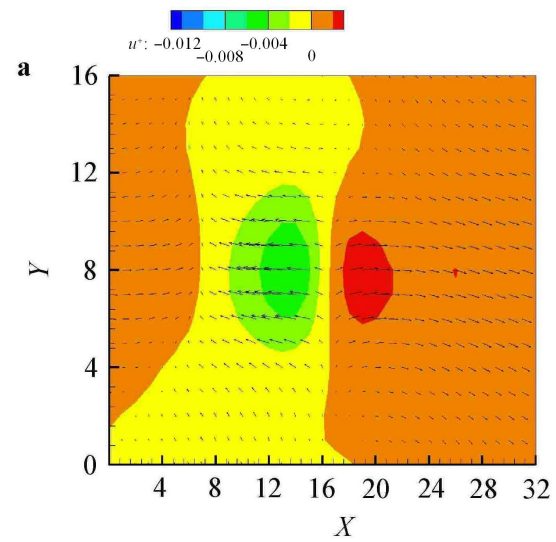

regular, indicating that the polymers make the flow field in the near-wall region less chaotic.

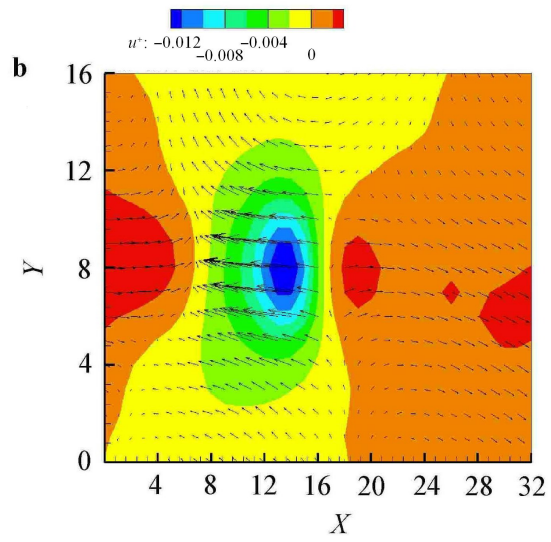

Fig. 8 Contours of streamwise fluctuating velocity during ejection. a PAM; b Water

The contours of the fluctuating velocity derivatives for the polymer solution and water during ejection are illustrated in Fig. 9, the left column figures of which are the characteristic quantities for the solution, and the right ones are all for water. Figures $9 \mathrm{a}$ and $9 \mathrm{~b}$ show the streamwise velocity gradients along the normal direction, given by $\mathrm{d} u_{12}=\partial u^{\prime} / \partial y$. It is obvious that in both figures there are antisymmetric structures, whose appearance is a universal feature of turbulent flow regeneration and sustaining. Compared with the streamwise velocity gradient along the wall-normal direction in water, the $\mathrm{d} u_{12}$ in the solution is reduced, indicating that the polymer additives slow the change of the coherent structures' streamwise velocity in the wall-normal direction. The normal velocity gradients along the streamwise direction, given by $\mathrm{d} u_{21}=\partial v^{\prime} / \partial x$, with and without polymers are presented in Figs. 9c and 9d. In these two figures the levels of contour legends are set to the same as those in Figs. 9a and $9 \mathrm{~b}$. It is found that there are hardly any spatial topologies in Figs. 9c and 9d, because the normal velocity gradients along the streamwise direction $\mathrm{d} u_{21}$ are much smaller than the streamwise velocity gradients along the normal direction $\mathrm{d} u_{12}$. And for the same reason, the spatial topology of velocity strain rates shown in Figs. 9e and 9f, defined as $s_{12}=\left(\partial u^{\prime} / \partial y+\partial v^{\prime} / \partial x\right) / 2$, are similar to that of the streamwise velocity gradients $\mathrm{d} u_{12}=\partial u^{\prime} / \partial y$. It is evident from the figure that the velocity strain rates becomes smaller under the action of the polymer additives. The decrease of the fluctuating velocities and velocity strain rate demonstrates that the polymers disrupt the energy transport of turbulent coherent structure. The energy conservation equation of coherent motion is given in Ref. [15] as

$$
\frac{\mathrm{d}}{\mathrm{d} t} \int \frac{\left\langle\tilde{u}_{i}^{\prime} \tilde{u}_{i}^{\prime}\right\rangle}{2} \mathrm{~d} V=-\int\left\langle\tilde{u}_{i}^{\prime} \tilde{u}_{j}^{\prime}\right\rangle\left\langle S_{i j}\right\rangle \mathrm{d} V+\int \tilde{r}_{i j} \tilde{s}_{i j} \mathrm{~d} V-\varphi_{c} .
$$

where, $\tilde{u}_{i}^{\prime}$ is the fluctuating velocity of the coherent structure, and $\tilde{s}_{i j}$ is the velocity strain rate. Term I and II on the righthand side of Eq. (8) represents the energy transport from the ensemble average movement and that to the coherent fluctuation, and Term III denotes the energy dissipation of molecular viscosity. For the turbulent flow with polymers, the fluctuating velocity declines compared with the case of water, which is obviously seen from Fig. 8. The modification by polymer additives of the velocity fluctuations, which contribute to the Reynolds shear stress, may affect the energy of coherent motion obtained from the ensemble average movement. Similarly, the velocity strain rate diminishes as shown in Figs. 9e and 9f, which leads to the decline of the energy cascade to the coherent fluctuation. Actually, the fact that the coherent fluctuation occupies less energy is partly responsible for the drag reduction. In this way, the polymer solution disrupts the energy transport of the coherent structure in wall turbulence, leading to the friction drag reduction. The spanwise vorticity components $\omega_{z}=\left(\partial v^{\prime} / \partial x-\partial u^{\prime} / \partial y\right) / 2$, which are the combination of $\mathrm{d} u_{12}$ and $\mathrm{d} u_{21}$, are presented in Figs. $9 \mathrm{~g}$ and $9 \mathrm{~h}$. The two figures resemble each other in shape, while they have a great difference in the magnitude. As can be seen from these figures, a strong vortex with positive vorticity caused by low velocity fluid ejection is below the center of ejection. A vortex with negative vorticity appears above the positive vorticity by induction effect. Another pair of counter-rotating vortexes is also generated by induction downstream, and their values are a little lower than the main vortex. The magnitude values of vorticity for polymer additive solution are much smaller than that of water, which is in accordance with the results of Kim et al. [25]. They found that the polymer additives suppressed the autogeneration of new vortices and vortex packet formation in dragreducing flows, by using dynamical simulations to track the evolution of hairpin vortices in turbulent channel flows. The long-chain polymers weaken the rotating motions via their viscoelastic property. 

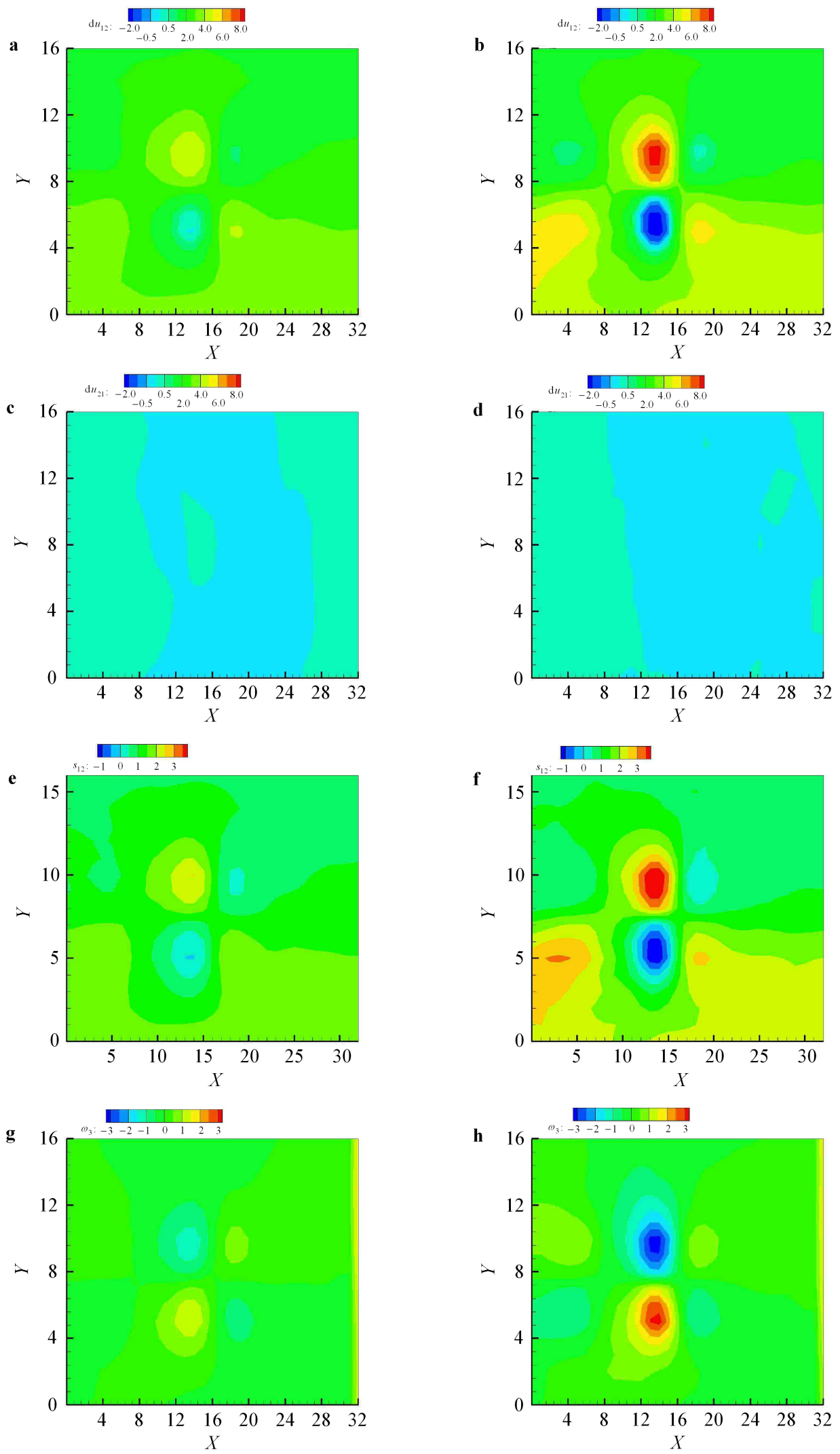

Fig. 9 Contours of velocity derivatives, velocity strain rate and vorticity during ejection: $\mathbf{a}$ and $\mathbf{b}$ velocity gradient $\mathrm{d} u_{12}=\partial u^{\prime} / \partial y ; \mathbf{c}$ and $\mathbf{d}$ Velocity gradient $\mathrm{d} u_{21}=\partial v^{\prime} / \partial x$; $\mathbf{e}$ and $\mathbf{f}$ velocity strain rate $s_{12}=\partial u^{\prime} / \partial y+\partial v^{\prime} / \partial x ; \mathbf{g}$ and $\mathbf{h}$ spanwise vorticity component. Left: PAM, right: water 


\section{Conclusions}

TRPIV measurements were performed in a water channel to investigate the structure of turbulent boundary layers with and without PAM at the same Reynolds number. Compared with the water case, the log-law profiles with the same slope in the drag reducing flow are displaced upward, which is associated with an increase in the thickness of the buffer layer. This effect reflects the character of drag reduction near the wall. Although the maximum value of streamwise velocity fluctuation is not affected by the viscoelasticity of polymers, the peaks of the wall-normal velocity fluctuation and the Reynolds shear stress are greatly reduced with polymer additives. In addition, the peaks of the turbulent statistics distribute broader in the wall-normal direction and shift farther away from the wall for the solution compared with the case of water. The solution with polymers reduces the contribution of the second and forth quadrants' fluctuations to the Reynolds shear stress, and suppress the fluid motion and the momentum transport. For this reason, the peak of turbulent kinetic energy becomes much smaller in the drag reducing flow. Moreover, the spatial topologies of coherent structures were extracted by using the new detection criteria which has advantages over the original one. Although the polymer additive solution does not affect the spatial topological shape of coherent structures in the wall turbulence, the fluctuating velocity and velocity derivatives of coherent structures burst drastically decreases in the solution. Because of the polymer additives, the wall friction is reduced by suppressing the occurrence and intensity of coherent structures, the dominant structures in near-wall turbulence.

\section{References}

1 Toms, B.A.: Some observations on the flow of linear polymer solutions through straight tubes at large Reynolds numbers. In: Proc 1st International Congress on Rheology, North Holland, 2, 135-141 (1948)

2 Virk, P.S., Merrill, E.W., Mickley, H.S., et al.: The Toms phenomenon: Turbulent pipe flow of dilute polymer solutions. J. Fluid Mech. 30, 305-328 (1967)

3 Lumley, J.L.: Drag reduction in turbulent flow by polymer additives. J. Polymer Sci. 7, 263-290 (1973)

4 Reischman, M.M., Tiederman, W.G.: Laser-Doppler anemometer measurements in drag-reducing channel flows. J. Fluid Mech. 70, 369-392 (1975)

5 Luchik, T.S., Tiederman, W.G.: Turbulent structure in lowconcentration drag-reducing channel flows. J. Fluid Mech. 190, 241-263 (1988)

6 Walker, D.T., Tiederman, W.G.: Turbulent structure in a channel flow with polymer injection at the wall. J. Fluid Mech. 218, 377-403 (1990)

7 Ptasinski, P.K., Nieuwstadt, F.T.M., Van Den Brule, B.H.A.A., et al.: Experiments in turbulent pipe flow with polymer additives at maximum drag reduction. Flow, Turbulence Combust 66, 159-182 (2001)
8 Oppong, F.K., Rubatat, L., Frisken, B.J., et al.: Microrheology and structure of a yield-stress polymer gel. Phys. Rev. E 73, 041405 (2006)

9 Donohue, G.L., Tiederman, W.G., Reischman, M.M.: Flow visualization of the near-wall region in a drag-reducing channel flow. J. Fluid Mech. 56, 559-575 (1972)

10 Achia, B.U., Thompson, D.W.: Structure of the turbulent boundary in drag-reducing pipe flow. J. Fluid Mech. 81, 439464 (1977)

11 Warholic, M.D., Heist, D.K., Katcher, M., et al.: A study with particle-image velocimetry of the influence of drag-reducing polymers on the structure of turbulence. Experiments in Fluids 31, 474-483 (2001)

12 Motozawa, M., Ishitsuka, S., Iwamoto, K., et al.: Experimental investigation on turbulent structure of drag reducing channel flow with blowing polymer solution from the wall. Flow, Turbulence Combust 88, 121-141 (2012)

13 Luchik, T.S., Tiederman, W.G.: Timescale and structure of ejections and burst in turbulent channel flows. J. Fluid Mech. 174, 529-552 (1987)

14 Jiang, N., Liu, W., Liu, J.H., et al.: Phase-averaged waveforms of Reynolds stress in wall turbulence during the burst events of coherent structures. Science in China Series G-Physics Mechanics and Astronomy 51, 857-866 (2008)

15 Zhang, Z.S., Cui, G.X., Xu, C.X.: Turbulence Theory and Simulation. Tsinghua University Press, Beijing (2005)

16 Kenis, P.R.: Turbulent flow friction reduction effectiveness and hydrodynamic degradation of polysaccharides and synthetic polymers. Journal of Applied Polymer Science 15, 607-618 (1971)

17 Cai, W.H.: Experiment study of drag-reducing characteristics for aqueous polyacrylamide solution. [M.S. Thesis], Harbin Institute of Technology, Harbin (2008)

18 Meza, J.C.: Steepest descent. Wiley Interdisciplinay Revies: Computational Statistics 2, 719-722 (2010)

19 Fan X., Jiang N.: Skin friction measurement in turbulent boundary layer by mean velocity profile method. Mechanics in Engineering 27, 28-30 (2005)

20 Warholic, M.D., Massah, H., Hanratty, T.J.: Influence of dragreducing polymers on turbulence: Effects of Reynolds number, concentration and mixing. Experiments in Fluids 27, 461-472 (1999)

21 Den Toonder, J.M.J., Hulsen, M.A., Kuiken, G.D.C., et al.: Drag reduction by polymer additives in a turbulent pipe flow: Numerical and laboratory experiments. J. Fluid Mech. 337, 193-231 (1997)

22 Wei, T., Willmarth, W.W.: Modifying turbulent structure with drag-reducing polymer additives in turbulent channel flows. J. Fluid Mech. 245, 619-641 (1992)

23 White, C.M., Somandepalli, V.S.R., Mungal M.G.: The turbulence structure of drag-reduced boundary layer flow. Experiments in Fluids 36, 62-69 (2004)

24 Liu, J.H., Jiang, N., Wang, Z.D., et al.: Multi-scale coherent structures in turbulent boundary layer detected by locally averaged velocity structure functions. Applied Mathematics and Mechanics 26, 495-504 (2005)

25 Kim, K., Adrian, R.J., Balachandar, S., et al.: Dynamics of hairpin vortices and polymer-induced turbulent drag reduction. Physical Review Letters 100, 134504 (2008) 